\title{
EBSD Sample Preparation: Techniques, Tips, and Tricks
}

\author{
Matthew M. Nowell*, Ronald A. Witt**, and Brian True* \\ * EDAX-TSL, 392 E 12300 S, Draper, UT 84020 \\ ** EBSD Analytical, 2044 N 1100 E, Lehi, UT 84043
}

One of the most common limitations on the performance of modern commercial EBSD systems is the quality of the pattern obtained after sample preparation. Often traditional metallographic sample preparation procedures include the use of etchants which preferentially attack grain boundaries to produce topographic contrast for observation. For EBSD, this topography is detrimental as it introduces intensity gradients within the EBSD patterns which can affect background correction and band detection. The EBSD patterns are formed from a depth of 10-50nm, and any residual surface deformation introduced during the preparation must be minimized in this region to maximize pattern quality. Typically standard metallography does not require such a fine surface finish and small final abrasive sizes. Additionally some etchants used leave a surface residue that is also detrimental to pattern quality. It should be noted that while these traditional methods can produce EBSD patterns that can be analyzed; the goal of EBSD sample preparation is to produce a flat deformation free surface that maximizes the pattern quality from the entire surface.

While every material can behave slightly differently during preparation, the basic concept of using progressively smaller polishing medium and finishing with a very small abrasive (approximately $0.05 \mu \mathrm{m}$ ) remains constant. Often it is easier to mount the sample to facilitate polishing. For EBSD analysis, a hot compressive conductive mount can make observation easier. However some samples cannot be subjected to heat or pressure. In these cases, cold mounting is more appropriate. SiC grinding papers are used for the initial grinding. Use as fine as steps are available between grit sizes $(240,320,400,600,800$, and 1200 for example) and minimize the amount of time with each step. Often these papers loose effectiveness after as little as 30 seconds. Diamond or alumina bases suspensions are often used for polishing in the $1 \mu \mathrm{m}$ and $0.25 \mu \mathrm{m}$ range. Colloidal silica (often $0.05 \mu \mathrm{m}$ ) makes an excelled final polishing medium for most materials. The combination of polishing and slight etching removes residual deformation well, and is often used in conjunction with a vibratory polisher. For ceramic and geological samples, the vibratory polishers can significantly affect pattern quality. When preparing multi-phase samples however, care must be used not to preferentially polish and remove phases.

Ion etching, either broad or focused beams, can also produce high quality EBSD patterns. This is often useful when other techniques are not possible or applicable.

[1] G. F. Vander Voort, Metallography Principles and Practice, ASM International, Ohio, 1999.

[2] D. Katrakova and F. Mucklich, Practical Metallographie, 38 (2001) 547.

[3] D. Katrakova and F. Mucklich, Practical Metallographie, 39 (2002) 644.

[4] M. L. Mulvihill et al., Zeitschrift fur Metallkunde, 89 (1998) 546.

[5] C. Boehlert et al., Scripta Materialia, 45 (2001) 1107.

[6] M.M. Nowell, Journal of Electronic Materials, 31 (2002) 23.

[7] S.V. Prasad et al., Scripta Materialia, 48 (2003) 255. 

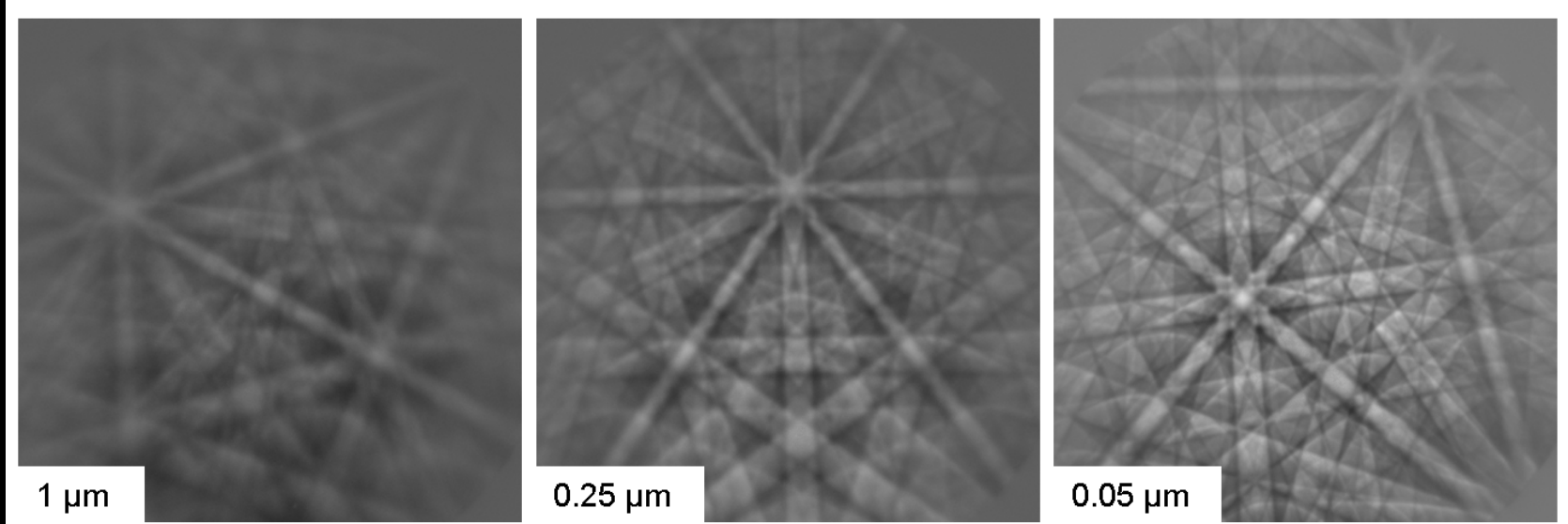

FIG. 1. EBSD patterns collected from Inconel 600. The sample was polished down to 1200 grit SiC followed by 10 minutes with $1 \mu \mathrm{m}$ diamond, $0.25 \mu \mathrm{m}$ diamond, and $0.05 \mu \mathrm{m}$ colloidal silica.

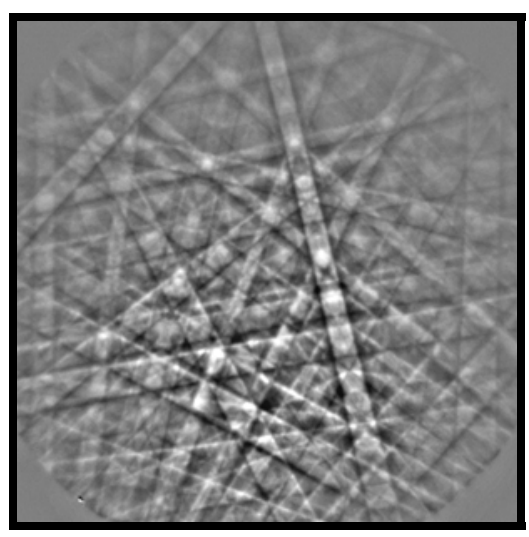

FIG. 2. EBSD pattern collected from SiC. The sample was polished down to $0.05 \mu \mathrm{m}$ colloidal silica with a vibratory polisher used for the final polish for 3 hours.

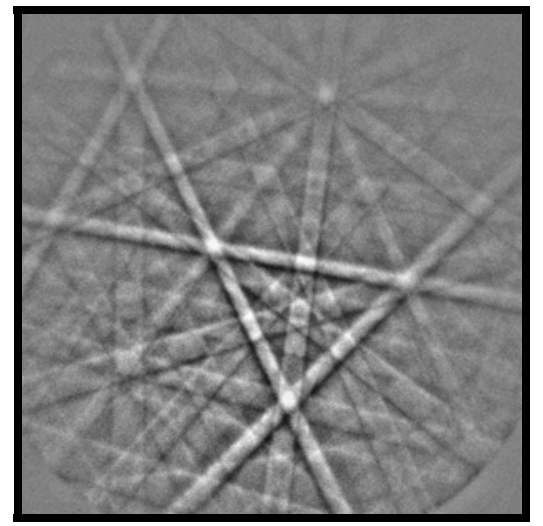

FIG. 3. EBSD pattern from an Mg alloy. The sample was polished down to 0.02 colloidal alumina with a vibratory polisher for 5 hours followed by an ion etching at $2.5 \mathrm{kV}$ for 8 hours. 